\title{
Comparing the impact of high pressure high temperature and thermal sterilization on the volatile fingerprint of onion, potato, pumpkin and red beet
}

\author{
Biniam T. Kebede, Tara Grauwet, Leonard Mutsokoti, Stijn Palmers, Liesbeth Vervoort, \\ Marc Hendrickx, Ann Van Loey* \\ Laboratory of Food Technology, Leuven Food Science and Nutrition Research Center (LFoRCe), Department of Microbial and Molecular Systems (M²S), KU Leuven, \\ Kasteelpark Arenberg 22 Box 2457, 3001 Heverlee, Belgium
}

\section{A R T I C L E I N F O}

\section{Article history:}

Received 4 November 2013

Accepted 20 December 2013

\section{Keywords:}

Vegetables

Thermal processing

High pressure high temperature processing

Sterilization

HS-SPME-GC-MS chemical fingerprinting

Process-induced chemical reactions

\begin{abstract}
A B S T R A C T
This work reports the effect of high pressure high temperature (HPHT) processing as compared to thermal processing on the volatile fraction of four, industrially-relevant, vegetables: onion, potato, pumpkin and red beet. The vegetables were selected from different botanical families, edible plant parts and color groups, aiming for investigation of a wide range of chemical reactions. Despite the difference in chemical composition among these vegetables, some remarkable general trends could be found. Firstly, in all vegetables, Strecker degradation products (such as 2- and 3methylbutanal) were detected at higher levels after HPHT sterilization in comparison to thermally sterilized counterparts. Secondly, HPHT sterilization enhanced oxidative degradation reactions (e.g. unsaturated fatty acids (in pumpkin, red beet and onion) and carotenoids (in pumpkin)). This work demonstrated the power of the untargeted multi-variate fingerprinting approach as a fast screening tool to zoom into relevant reaction pathways out of a complex network of chemical changes and to determine discriminative volatiles which can serve as potential markers. (c) 2013 Elsevier Ltd. All rights reserved.
\end{abstract}

\section{Introduction}

From all emerging food processing technologies which have been investigated as alternatives to the conventional thermal processing, in the context of wet food processing, high pressure can be labeled as the most studied and developed one. This novel technique has already shown a fast progression from the research and development environment into widespread commercial applications. In this context, high pressure processing has successfully been introduced at an industrial scale for pasteurizing a large range of food products, e.g. fruit juices, guacamole, oysters and ham (Knorr et al., 2011; Mujica-Paz, Valdez-Fragoso, Samson, Welti-Chanes, \& Torres, 2011).

Recently, the use of high pressure processing for sterilization of foods has been the focus of intensive studies (although without industrial implementation so far). The sterilization intensity is realized through an innovative approach that exploits the effect of pressures above or equal to $600 \mathrm{MPa}$, accompanied with elevated process temperatures $\left(90-121^{\circ} \mathrm{C}\right)$ : a processing method called high pressure high temperature (HPHT). As described by the principle of Pascal, pressure

\footnotetext{
* Corresponding author. Tel.: 01632 15 67; fax: 016321960

E-mail addresses: BiniamTamiru.Kebede@biw.kuleuven.be (B.T. Kebede), Ann.Vanloey@biw.kuleuven.be (A. Van Loey).

URL's: http://www.biw.kuleuven.be/m2s/clmt/lmt/ (B.T. Kebede),

http://www.biw.kuleuven.be/m2s/clmt/lmt/staff/current/permanent/ann-van-loey (A. Van Loey).
}

is transmitted instantaneously and uniformly. This phenomenon creates a rapid temperature change (due to the compression heating), leading to smaller come-up times in food products and thus to overall shorter processing times. In that context, one of the unique advantages of this novel sterilization technique is the possibility to apply the hightemperature short-time principle for conductive-heating foods (de Heij et al., 2003; Grauwet, Van der Plancken, Vervoort, Hendrickx, \& Van Loey, 2011; Knorr et al., 2011; Matser, Krebbers, van den Berg, \& Bartels, 2004; Meyer, Cooper, Knorr, \& Lelieveld, 2000; Wilson, Dabrowski, Stringer, Moezelaar, \& Brocklehurst, 2008).

In the last two decades, the knowledge about HPHT processing has been increasing. For instance, some answers were already given with respect to insight and control of relevant processing parameters in the context of up-scaling. For example, for temperature uniformity mapping, the use of computational thermal fluid dynamics modeling and simulation of temperature fields (Rauh, Baars, \& Delgado, 2009) and use of pressure-temperature-time indicators (Grauwet et al., 2011; Grauwet et al., 2012) were investigated. In addition, the impact on microbial safety is well studied, where in most cases HPHT processing is linked with a comparable or more effective inactivation of spores and pathogenic microorganisms at a particular process temperature and time (Ahn, Balasubramaniam, \& Yousef, 2007; Ramaswamy, Shao, \& Zhu, 2010; Reineke, Mathys, Heinz, \& Knorr, 2008; Wilson et al., 2008; Yanwen, Songming, Ramaswamy, \& Marcotte, 2010). However, with respect to the effect on quality and chemical safety of plant-based foods, even though there is a progress (De Roeck, Sila, Duvetter, Van Loey, \& 
Hendrickx, 2008; De Vleeschouwer, Van der Plancken, Van Loey, \& Hendrickx, 2010; Kebede et al., 2013; Oey, Lille, Van Loey, \& Hendrickx, 2008; Van der Plancken et al., 2012; Vervoort, Van der Plancken, et al., 2012), the knowledge is far from being fully documented. The major stumbling blocks in relation to that are the limited information about the unique effect of high pressure on chemical changes (Le Chatelier principle) and the incompletely understood synergistic/antagonistic effect of high pressure with increased temperature. In the context of pressure on quality reactions, no general effect seems to persist, in which the research results from most of the studies showed that the effect depends on the reaction type and differs from matrix to matrix. Therefore, there is a need for screening different food matrices to add depth into our understanding about the effect of HPHT processing on important quality-related chemical changes.

The present work aims to investigate and compare process-induced chemical changes in HPHT and conventional thermally sterilized vegetables. To get a deeper understanding on a wide range of reaction pathways, the comparison was performed on four commercially-relevant vegetables, i.e. onion, potato, pumpkin and red beet, which were selected from different botanical families, type of edible plant parts and color groups. The vegetables were sterilized with thermal and HPHT processing that resulted in equivalent microbial safety.

In literature, many possible approaches have been exploited to compare the impact of processing technologies, such as targeted and untargeted. In this work, an untargeted approach was used as a hypothesis-free screening technique to compare the volatile fraction of differently processed vegetables. Semi-volatile and volatile compounds are among the common end-products of many process-induced chemical reactions, and can be a witness for what is happening not only in the volatile but also in the other fractions. Consequently, analyzing the volatile fraction can be considered as a good starting point for comparing processing impacts.

Process-induced chemical reactions are mostly complex and with different reaction pre-cursors and intermediates interacting. In addition, the knowledge about these interactions is limited and can even get more complicated since HPHT processing could affect reactions differently and/or induce some new pathways. This makes the process of identifying and characterizing important chemical reactions less straightforward. In such complex network of reactions, focusing on a particular reaction pathway or reaction product from the beginning (which is a targeted approach) might result in overlooking some beneficial/detrimental changes. Therefore, in this work, an (MS)-based chemical fingerprinting platform was implemented to detect as many as possible volatile compounds in the headspace fraction of processed vegetable puree. This means the approach doesn't focus on a single reaction pathway but takes into consideration almost all chemical changes responsible for the formation of semi-volatile and volatile compounds (which is an untargeted multi-variate approach). In this platform, headspace-solid phase microextraction (HS-SPME) was integrated with gas chromatography (GC) and MS detection. The huge amount of data generated by GC-MS is analyzed using chemometric tools (e.g. partial least squares discriminant analysis (PLS-DA)).

\section{Materials and methods}

\subsection{Sample preparation}

Fresh vegetables: a single batch of pumpkin (Cucurbitaceae), red beet (Amaranthaceae), onion (Alliaceae) and potato (Solanaceae) were purchased at a local market. Since these vegetables were selected from different botanical families, type of edible plant parts and color groups, in this work, different mixes of natural pre-cursors of reactions were taken into account. The vegetables were carefully washed and cut into standardized pieces. The pumpkin, red beet and potato were cut into cylinders (approximately $1 \mathrm{~cm}$ thickness) and the onions were cut into $1 \mathrm{~cm}$ thick slices. The vegetables were then put into low density polyethylene bags. To prevent all enzymatic reactions during processing, storage and thawing, the vegetables were blanched at $95^{\circ} \mathrm{C}$ for $8 \mathrm{~min}$ in a water bath (Haake W15 DC-10, Germany). The blanched plastic bags were immediately cooled in ice water for $10 \mathrm{~min}$, frozen in liquid nitrogen and stored in a freezer at $-40{ }^{\circ} \mathrm{C}$. The blanching conditions were validated using a qualitative and quantitative peroxidase (POD) test (Adebooye, Vijayalakshmi, \& Singh, 2008; Vervoort, Van der Plancken, et al., 2012). Prior to processing, the samples were thawed overnight at $4{ }^{\circ} \mathrm{C}$. A Buchi mixer (B-400, BUCHI, Switzerland) was used to blend the blanched vegetables.

\subsection{Technological processing}

With the aim of having a fair impact comparison, the processing conditions in both thermal and HPHT sterilization were selected, targeting an equivalent inactivation of spores of Clostridium botulinum $\left(F_{121.1}^{10}{ }^{\circ} \mathrm{C}\left(F_{0}\right)=5 \mathrm{~min}\right)$. A holding temperature $\left(T_{h}\right)$ of $117^{\circ} \mathrm{C}$ was selected for both processing treatments. Due to the lack of reliable kinetic data as a result of incomplete understanding of the combined effect of pressure and temperature (Van der Plancken et al., 2012), in the present work, the HPHT treatment was considered as pressure assisted thermal treatment. In other words, for calculating the $F_{0}$ value, only the integrated thermal impact (from the time-temperature profiles) was taken into account. Each treatment category was repeated 6 times. The profiles of the recorded process variables for both thermal and HPHT treatments are depicted in Kebede et al. (2013). Even though, the profile on our previous study was for different matrices (spinach, green pepper and broccoli), due to the high water content in all puree, the profiles were comparable. Due to their inert nature, glass jars for the thermal processing and Teflon sample holders for the HPHT processing were selected.

\subsubsection{Thermal processing}

The thermal treatment was carried out in a static steriflow pilot retort (Barriquand, Paris, France). The glass jars (100 ml volume, $95 \mathrm{~mm}$ height and $45 \mathrm{~mm}$ diameter) were filled with $85 \pm 0.5 \mathrm{~g}$ of vegetable puree and then closed with metal lids. Next, they were loaded into the retort and sterilized for a processing time of $80 \mathrm{~min}$. Temperature profiles in the retort and at the coldest point of the product were recorded using type $\mathrm{T}$ thermocouples (Ellab, Denmark).

\subsubsection{High pressure high temperature processing}

The HPHT treatment was carried out in laboratory-scale HPHT equipment (custom-made, Resato, The Netherlands). The equipment consists of six vertically oriented individual vessels (volume $=43 \mathrm{~cm}^{3}$ and diameter $=2 \mathrm{~cm}$ ). The vessels were jacketed with a heating coil connected to a temperature controlling unit. The HPHT equipment allows a computer-controlled pressure build-up of up to $800 \mathrm{MPa}$, temperature control of up to $120{ }^{\circ} \mathrm{C}$ and data logging of both sample pressure and temperature. The pressure medium was $100 \%$ propylene glycol (PG fluid, Resato, The Netherlands). During a HPHT treatment, a preheating at atmospheric pressure, pressure build-up, and holding and cooling steps were established (Grauwet, Van der Plancken, Vervoort, Hendrickx, \& Van Loey, 2010; Grauwet et al., 2012; Kebede et al., 2013; Van der Plancken et al., 2012).

Teflon (polytetrafluoroethylene) sample holders (12 mm inner diameter, $85 \mathrm{~mm}$ length, $4 \mathrm{~mm}$ wall thickness, Vink, Belgium) were filled with vegetable puree and closed with a movable cap and vacuum sealed with double plastic bags. The sample holders were pre-equilibrated at $10{ }^{\circ} \mathrm{C}$ in a cryostat after which they were loaded into the HP vessels that were equilibrated at $117^{\circ} \mathrm{C}$. Starting from room temperature, using only compression heating, the product temperature cannot be raised to the point where inactivation of spores under high pressure is feasible. Therefore, prior to the actual HPHT treatment, the samples were preheated at atmospheric pressure to experimentally determined the initial temperature $\left(T_{i}\right) \sim 75^{\circ} \mathrm{C}$. When the desired $T_{i}$ was achieved, pressure in the vessels was increased through indirect compression. 
During the pressure build-up, two consecutive stages can be identified: (i) instantaneous pressure increase from 0.1 to $150 \mathrm{MPa}$; (ii) further pressure increase until $600 \mathrm{MPa}$ at a rate of $10 \mathrm{MPa} / \mathrm{s}$. After reaching $600 \mathrm{MPa}$ and an equilibration time of $1 \mathrm{~min}$ was taken into account, the individual vessels were isolated. Due to the pressurization and isolation processes, the temperature inside the product was increased from $T_{i}$ to $117{ }^{\circ} \mathrm{C}$ through compression heating. The product temperature was recorded online and the holding time was adjusted to achieve the targeted $F_{0}$ value. On average, the pressure was held for $15 \mathrm{~min}$. At the end of the holding time, the pressure was released from the vessels, which was accompanied with temperature drop inside the product (decompression cooling).

\subsection{Post treatment sample handling}

Following treatments, samples were immediately transferred to ice water to stop any further reaction. Consequently, treated samples were emptied in a cooling room $\left(4{ }^{\circ} \mathrm{C}\right)$ and transferred to small volume $(10 \mathrm{ml})$ polyethylene terephthalate tubes with a polyethylene cap. Hereafter, the tubes were frozen in liquid nitrogen and stored at $-40{ }^{\circ} \mathrm{C}$ until analysis.

\subsection{HS-SPME-GC-MS analysis}

Samples were thawed overnight in the cooling room $\left(4^{\circ} \mathrm{C}\right) .2 .5 \mathrm{~g}$ of the thawed sample and $2.5 \mathrm{ml}$ of saturated $\mathrm{NaCl}$ solution were mixed into a $10 \mathrm{ml}$ amber glass vial $(10 \mathrm{ml}$, VWR International, Radnor, USA). The vials were tightly closed using screw-caps with PTFE/silicon septum seal (GRACE, Columbia, MD, USA), homogenized and transferred to the cooling tray of the auto-sampler which was maintained at $10{ }^{\circ} \mathrm{C}$. Headspace fingerprinting was conducted on a gas chromatography (GC) system (6890N, Agilent Technologies, Diegem, Belgium) coupled to a mass selective detector (MSD) (5973N, Agilent Technologies, Diegem, Belgium) and equipped with a CombiPAL autosampler (CTC analytics, Zwingen, Switzerland). Targeting detection of a wide range of volatiles in a particular food extract, a HS-SPME-GC-MS method of analysis was optimized for each matrix beforehand. The method includes incubation and extraction using an appropriate type of fiber coating and GC-MS parameters. In the selected method, the samples were incubated at $40{ }^{\circ} \mathrm{C}$ for 20 min under agitation at $500 \mathrm{rpm}$. Next, extraction of the volatiles from the headspace was performed using HS-SPME fiber coated with 30/50 $\mu$ m divinylbenzene/carboxen/ polydimethylsiloxane (DVB/CAR/PDMS) (StableFlex, Supelco, Bellefonte, PA, USA) at $40{ }^{\circ} \mathrm{C}$ for $10 \mathrm{~min}$. The SPME fiber was inserted into the heated $\left(230{ }^{\circ} \mathrm{C}\right) \mathrm{GC}$-injection port for 2 min to desorb the volatile compounds. Prior to extraction, the fibers were conditioned and regenerated according to the manufacturer's guidelines in the conditioning station of the auto-sampler. Injection of the samples to the GC-column was performed in split (1/5) mode. Chromatographic separation was carried out on an HP-5MS capillary column (30 m $\times 0.25 \mathrm{~mm}$ i.d., $0.25 \mu \mathrm{m}$ film thickness, Agilent Technologies J\&W, Santa Clara, CA, USA) having 5\%-phenylmethylpolysiloxane as a stationary phase and helium as a gas phase at a constant flow of $1.5 \mathrm{ml} / \mathrm{min}$. This non-polar column is appropriate for trace level analyses, e.g. semi-volatiles and volatiles. The GC-oven temperature was programmed from a starting temperature of $40{ }^{\circ} \mathrm{C}$, which was retained for $2 \mathrm{~min}$, to $172{ }^{\circ} \mathrm{C}$ at $4{ }^{\circ} \mathrm{C} / \mathrm{min}$, then ramped to $300{ }^{\circ} \mathrm{C}$ at $30{ }^{\circ} \mathrm{C} / \mathrm{min}$ and kept constant at $300{ }^{\circ} \mathrm{C}$ for 2 min before cooling back to $40{ }^{\circ} \mathrm{C}$. The mass spectra were obtained by electron ionization (EI) mode at $70 \mathrm{eV}$ with a scanning range of 35 to $400 \mathrm{~m} / \mathrm{z}$ and a scanning speed of 3.8 scans/s. MS ion source and quadrupole temperatures were $230{ }^{\circ} \mathrm{C}$ and $260{ }^{\circ} \mathrm{C}$, respectively.

\subsection{Data pre-processing and multivariate analysis}

As commonly observed in GC-MS analysis, co-eluting compounds were present in the obtained chromatograms. Therefore, all chromatograms were analyzed with an Automated Mass Spectral De-convolution and Identification System (AMDIS) (Version 2.66, 2008, National Institute of Standards and Technology, Gaithersburg, MD, USA) to extract "pure" component spectra from complex chromatograms. In addition, AMDIS can be configured to build a retention index calibration file and to use retention index data for proof of identity along with the mass spectral data. The de-convoluted spectra were then analyzed with Mass Profiler Professional (MPP) (Version 12.0, 2012, Agilent Technologies, Diegem, Belgium) aiming for filtering and peak alignment. The MPP obtained a spreadsheet containing peak areas, which was used as an input for the statistics. The multivariate data were analyzed with a multivariate data analysis (MVDA) which was carried out in Solo (Version 6.5, 2011, Eigenvector Research, Wenatchee, WA, USA). These advanced data analysis techniques allow visualizing and investigating the massive data by reducing the dimensionality to a few dimensions only. As a preprocessing step, all data were mean-centered and the variables were weighed by their standard deviation to give them equal variance. To compare the treatment impact, a regression-based supervised classification technique, namely partial least squares discriminant analysis (PLS-DA) was implemented. For PLS-DA, the volatile compounds were considered as $X$-variables and the three classes, blanched (reference), thermal processing and HPHT processing, were considered as categorical $Y$ variables. Determining the complexity of the model, the lowest number of latent variables (LVs) resulting in a class separation were used. In PLS-DA, to investigate impact differences among the classes, bi-plots were plotted. To select discriminant volatile compounds, Variable IDentification (VID) coefficients were calculated (Vervoort, Grauwet, et al., 2012; Kebede et al., 2013). These values correspond to the correlation coefficient between each original $X$-variable and $Y$-variable (s). Variables with an absolute VID value higher than 0.800 were considered to be important. These discriminant volatiles were plotted individually as a function of processing. In these plots, the mean areas and the standard errors calculated from the six replicates were depicted. All plots were made using OriginPro 8 (Origin Lab Corporation, Northampton, MA, USA). A Duncan's multiple comparison was used to test for significant differences between the mean peak areas $(p<0.05)$ of the discriminant volatiles. Identification of these compounds was performed by comparing the deconvoluted mass spectrum with the reference mass spectra from both NIST spectral library (NIST08, version 2.0, National Institute of Standards and Technology, Gaithersburg, MD, USA) and WILEY registry of mass spectral data (Wiley 2010, version 9, USA). A threshold match of $90 \%$ was implemented and for confirmation further visual inspection of the spectral matching was conducted.

\section{Results and discussion}

Food components that take part in chemical reactions vary depending on the type of matrices. In that context, aiming to screen a broad range of chemical changes, vegetables from different botanical families, edible plant parts and color groups were selected: onion, potato, pumpkin and red beet. To have a fair comparison between novel and conventional food processing technologies, the vegetables were HPHT and thermal sterilized targeting an equivalent process value. Next, an MSbased metabolic fingerprinting (HS-SPME-GC-MS) method was implemented to analyze and compare the volatile fractions of sterilized vegetables. Examples of obtained total ion chromatograms for each (blanched) vegetable are shown in Fig. 1.

Even though GC-MS provides a good compound separation, the chromatograms commonly contain extraneous mass spectral peaks. These extraneous peaks can pose a serious problem during compound identification. Therefore, prior to the multivariate statistical data analysis (MVDA), two consecutive data pre-processing techniques were conducted on the raw GC-MS data. First, the co-eluted peaks were de-convoluted by the Automated Mass Spectral De-convolution and Identification System (AMDIS). Next, Mass Profiler Professional (MPP) was used to filter and align peaks. 

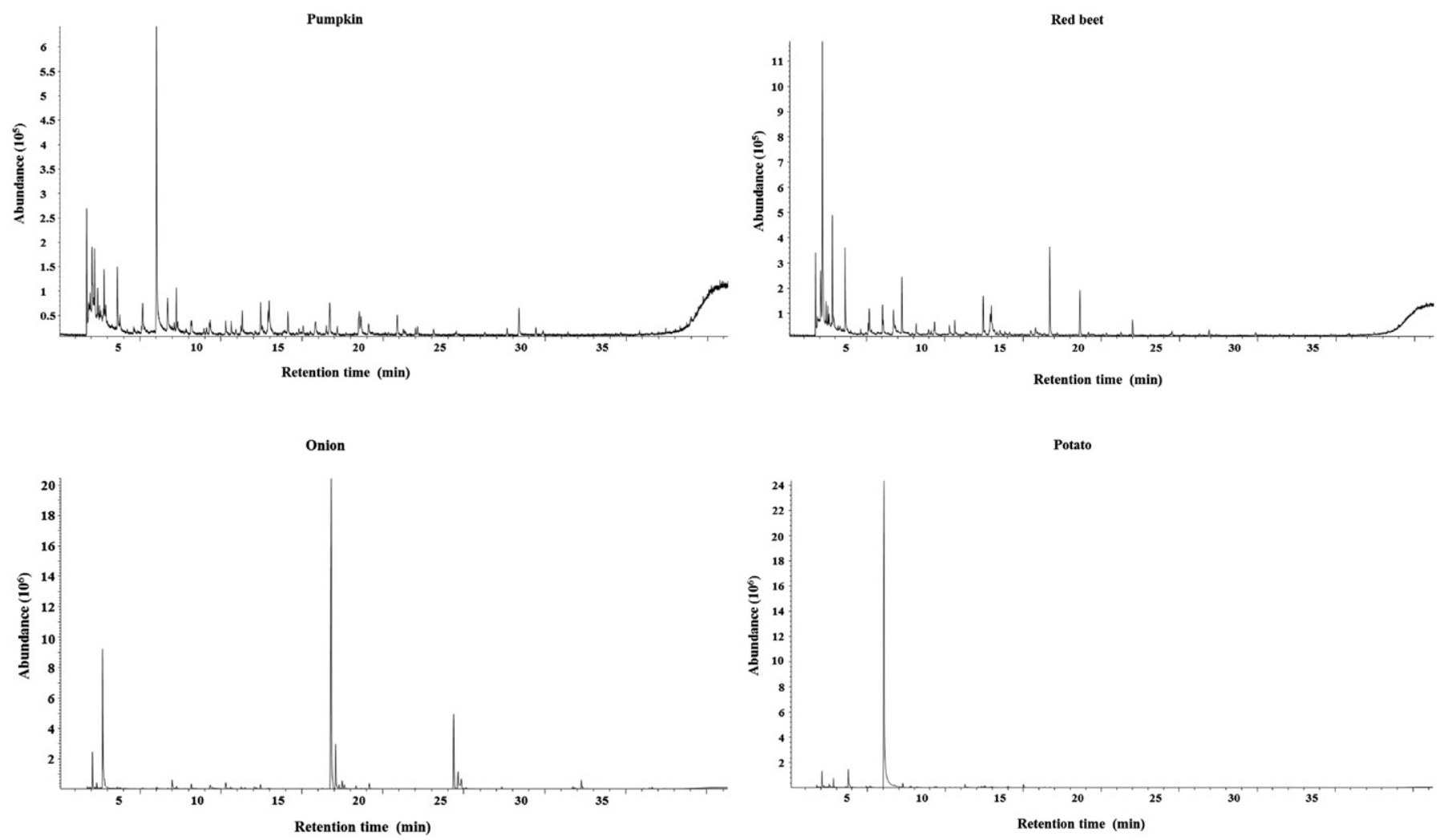

Fig. 1. Total ion chromatograms of a volatile fraction of blanched vegetables analyzed by HS-SPME-GC-MS.

Next, per vegetable, a partial least squares discriminant analysis (PLS-DA) model was built using two latent variables (LVs), in which the respective bi-plots are shown in Fig. 2 . The LVs are nothing but the linear combination of the original $X$-variables (volatiles) for which the separation among the $Y$-variables (blanched, thermal and HPHT classes) is maximum. As can be seen from the bi-plots, per vegetable, only $2 \mathrm{LVs}$ described more than $93 \%$ of the variance among the classes, demonstrating a clear different effect of the applied processing technologies on the volatile fraction of the vegetables. On each plot, there are three vectors, representing the importance of the selected LVs for class separation (i.e. PLS-DA model performance). If proper numbers of LVs are selected for the model, the vectors will be long and pointing to the direction of the respective class (which is the case in Fig. 2). The biplots also provide qualitative information about the importance of the volatiles for the selection of LVs and consequently for class separation. For instance, if the concentration of a certain volatile is increased by HPHT sterilization compared to thermal sterilization, on the plot, this compound should be located close the HPHT class and vice versa. However, it is relevant to quantify the individual effect of the volatiles for class separation, in other words identify casual variables. In that sense, the contribution of each volatile compounds for class separation is nothing else than the correlation between the volatile compound and the classes, which is termed as the VID procedure. Accordingly, VID coefficients were calculated to rank volatiles based on their concentration in one class compared to another one. Per class, each volatile was assigned with a value between -1 and +1 , where a positive VID coefficient represents a higher concentration in that class compared to other classes and vice versa. Compounds with an absolute VID value higher than 0.800 were considered to be important and were selected as discriminant volatiles (Table 1; Fig. 2 (bold open circles)). The presence of a significant difference $(\mathrm{p}<0.05)$ (see Section 2.5) between the different classes was used as a criteria to determine the above cut-off/VID threshold value. The compounds that passed this criterion were identified and plotted individually as a function of processing (results not shown). To put focus on the most discriminative ones, those with a total peak area difference of $\geq 90 \%$, compared to at least one of the other classes, are put in italic. In addition, compounds detected only in one class, which can be potential markers, are put in bold italic. As can be seen from Fig. 2 and Table 1, in all vegetables, almost all discriminant volatiles are in italic, meaning their concentration is more than $90 \%$ different in the different classes. In addition, 3, 1, 8 and 9 volatiles were detected as potential markers in pumpkin, red beet, onion and potato, respectively.

Even though studies reporting the effect of high pressure at elevated temperature on the formation of volatile compounds in pumpkin, red beet, onion and potato are scarce, in the following sections (Sections 3.1-3.4), vegetable by vegetable, it will be tried to link the selected discriminant volatiles to possible reaction pathways, since this will increase insight into the effect of this novel food sterilization technique on quality-related chemical reactions.

\subsection{Pumpkin}

Pumpkin is a good source of beta-carotene, water-soluble vitamins and amino acids (Seo, Burri, Quan, \& Neidlinger, 2005). Processed pumpkin has a characteristic volatile profile comprising of aldehydes, ketones, alcohols, furan derivatives, sulfur-containing compounds and N-hetero-cyclic compounds (Siegmund \& Murkovic, 2004). The formation of these volatile compounds is mostly linked to chemical reactions, such as carotenoid degradation, lipid degradation and the Maillard reaction. Thermal-induced degradation of carotenoids leads to the formation of compounds such as 2-hydroxy-2,6,6-trimethylcyclohexanone, 2,6,6-trimethylcyclohexanone, 2-hydroxy-2,6,6-trimethylcyclohexane-1-carboxaldehyde and 2,6,6-trimethyl-2-cyclohexen-1-one (Kanasawud \& Crouzet, 1990). As can be seen in Table 1, such compounds (e.g. 2,2,6-trimethylcyclohexanone, $\alpha$-ionone, 1,2,5,5-tetramethyl-1,3cyclopentadiene, $\alpha$-cyclocitral, 2,6,6-trimethyl-1-cyclohexene-1-acetaldehyde) were dominantly selected discriminating the volatile fraction of HPHT and conventional thermally treated pumpkin puree. These compounds were detected at higher concentration after HPHT sterilization, 

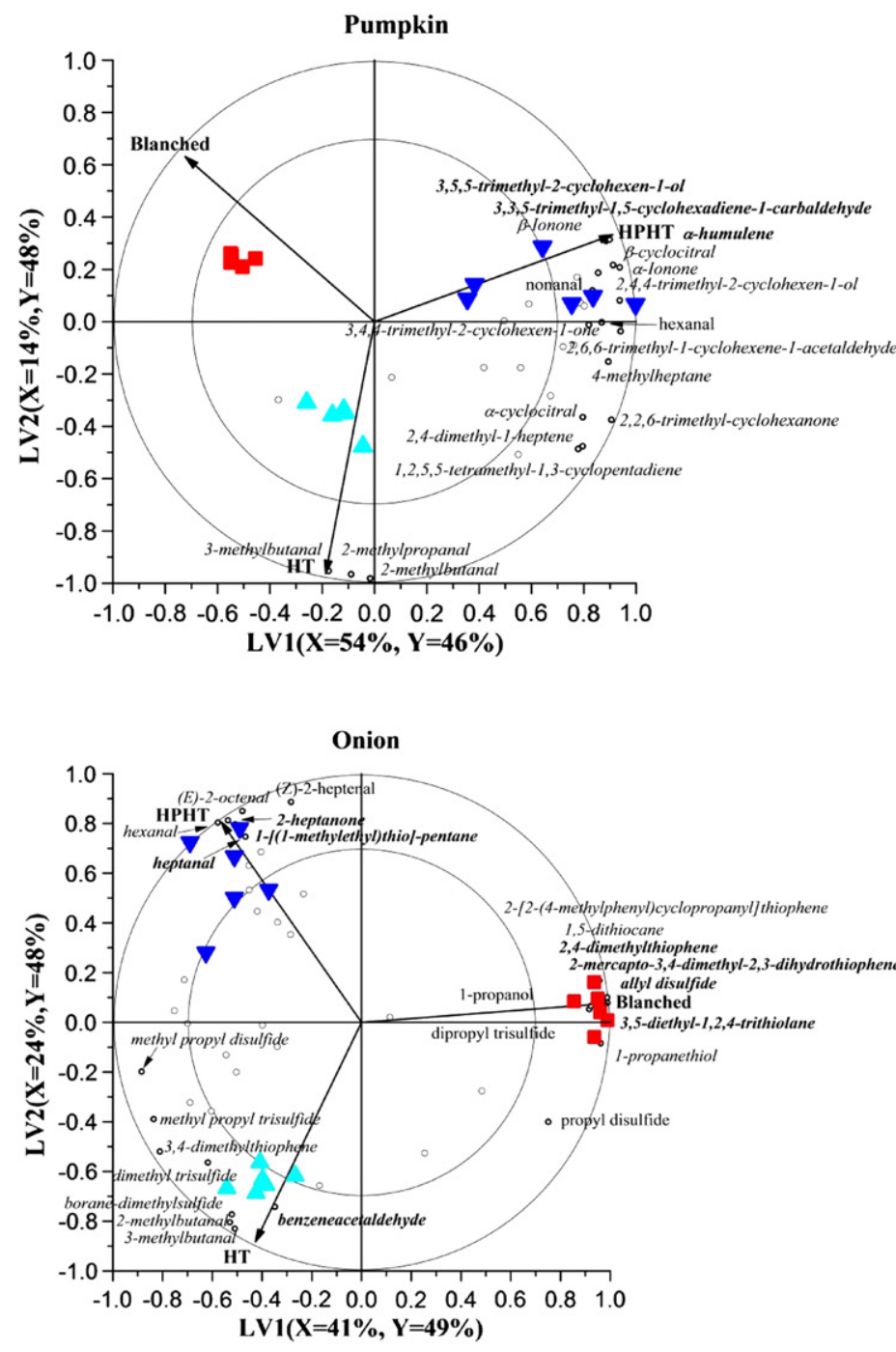

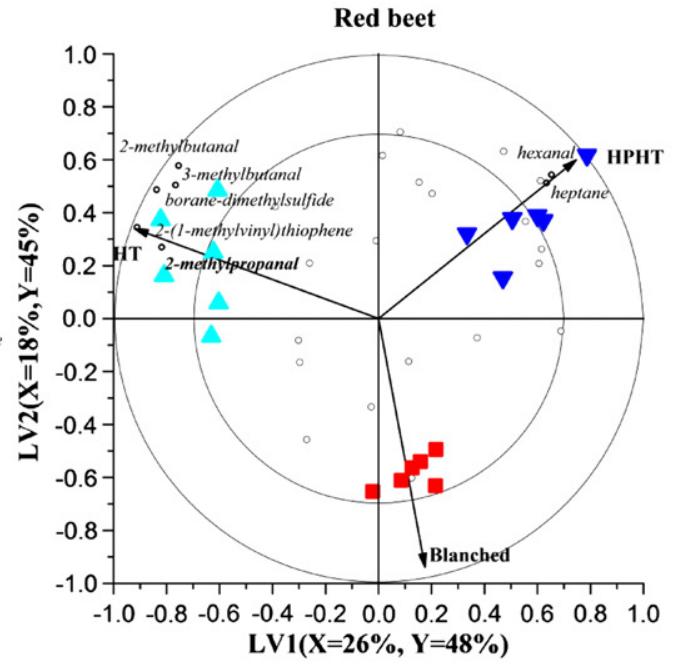

Potato

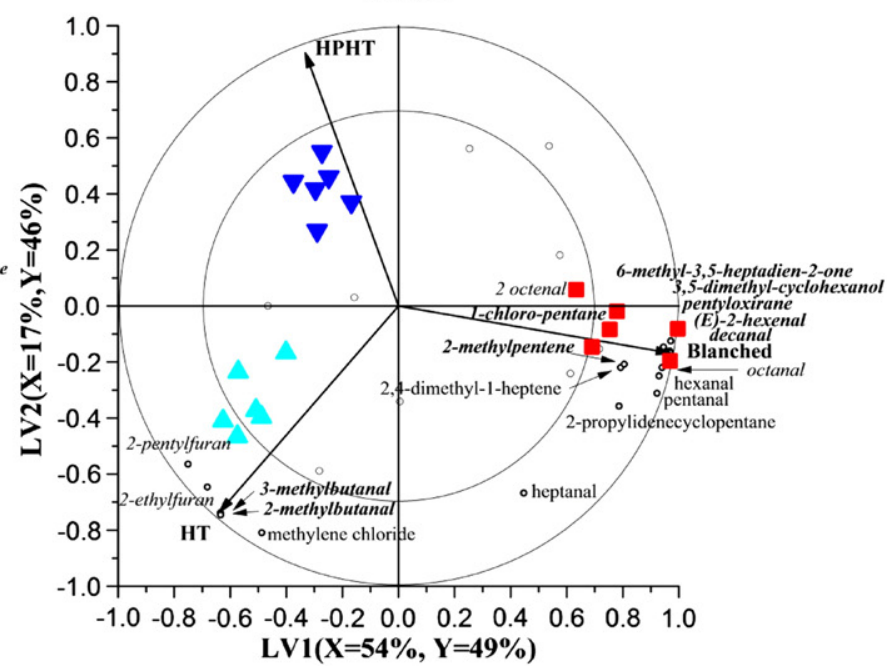

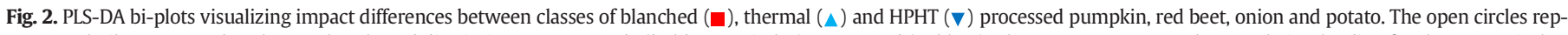

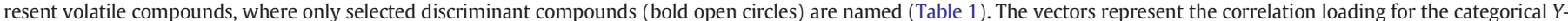

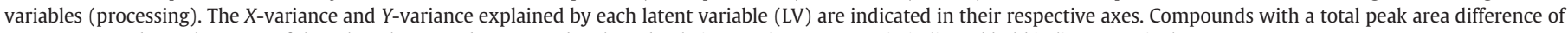
$\geq 90 \%$ compared to at least one of the other classes and compounds selected only in one class were put in italic and bold italic, respectively.

i.e. HPHT sterilization is inducing an increased formation of (oxidative) carotenoid degradation products compared to its thermal conventional counterpart. This means that the concerted effect of the processing variables ( $p, T, t)$ was stronger than the concerted effect of $T$ and $t$ under the studied conditions. The second group of chemical compounds that are selected as discriminant volatiles are aliphatic aldehydes, such as hexanal and nonanal, which are mostly linked with degradation of unsaturated fatty acids. The concentration of these compounds was remarkably increased by HPHT sterilization compared to equivalent conventional thermal sterilization. An increased formation of the aliphatic aldehydes under high pressure was also previously reported in other studies (Navarro, Verret, Pardon, \& EL Moueffak, 2002; Oey, Lille, Van Loey, \& Hendrickx, 2008; Oey, Van der Plancken, Van Loey, \& Hendrickx, 2008; Porretta, Birzi, Ghizzoni, \& Vicini, 1995). It is noteworthy that both carotenoid and unsaturated fatty acid degradation can be linked to oxidative reactions, which seem to be favored by high pressure. This is in line with reports in which oxidative chemical reactions were enhanced under increased pressure (Kebede et al., 2013; Oey, Van der Plancken, Van Loey, \& Hendrickx, 2008; Van der Plancken et al., 2012; Verbeyst, Bogaerts, Van der Plancken, Hendrickx, \& Van Loey, 2012). Finally, Strecker aldehydes, such as 2-methylbutanal, 2-methylpropanal and 3methylbutanal, were detected with a significantly higher amount after thermal sterilization compared to HPHT sterilization. These compounds are reaction products of one of the side reactions of the Maillard reaction (i.e. the Strecker degradation). Accordingly, the research result in this work indicates that the Maillard reaction seems to be more enhanced by conventional thermal processing compared to an equivalent HPHT processing or in other words the reaction is suppressed by HPHT sterilization. This observation is in agreement with the findings in previous studies (De Vleeschouwer et al., 2010; Hill, Isaacs, Ledward, \& Ames, 1999; Kebede et al., 2013; Vervoort et al., 2013).

\subsection{Red beet}

Red beet is a traditional and popular vegetable in many parts of the world. It is especially rich in fibers as well as in sugars but with moderate caloric value. The soluble and cell wall associated phenolics and betalains are the main pigments in red beet that are responsible for its reddish-purple hue and are also known to be bioactive compounds (Schwartz \& Vonelbe, 1980). From Table 1, few discriminative volatiles were selected in red beet compared to the other vegetables. It seems that red beet is characterized with a very poor volatile fraction. This can be explained by the fact that betalains are high molecular weight molecules which are not easily degraded into short chain compounds. 
Table 1

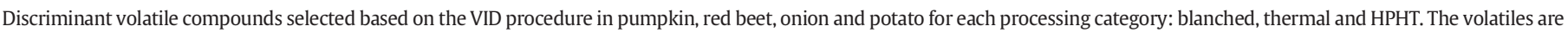

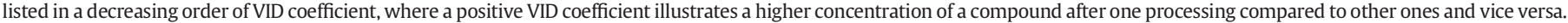

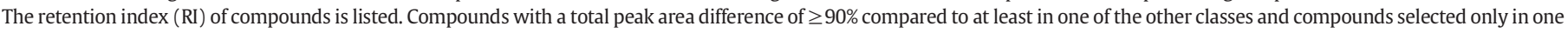
class were put in italic and bold italic, respectively.

\begin{tabular}{|c|c|c|c|c|c|c|c|c|c|}
\hline & \multicolumn{3}{|l|}{ Blanched } & \multicolumn{3}{|c|}{ Thermal processing } & \multicolumn{3}{|c|}{ HPHT processing } \\
\hline & VID & Identity & RI & VID & Identity & RI & VID & Identity & RI \\
\hline \multirow[t]{20}{*}{ Pumpkin } & -0.980 & 2,2,6-Trimethylcyclohexanone & 1035 & & & & & & \\
\hline & -0.921 & 2,4-Dimethyl-1-heptene & 846 & & & & & & \\
\hline & -0.910 & 1,2,5,5-Tetramethyl-1,3-cyclopentadiene & 848 & & & & & & \\
\hline & -0.878 & 4-Methylheptane & 788 & & & & & & \\
\hline & -0.876 & $\alpha$-Cyclocitral & 1119 & & & & & & \\
\hline & -0.873 & $\begin{array}{l}\text { 2,6,6-Trimethyl-1-cyclohexene-1- } \\
\text { acetaldehyde }\end{array}$ & 1261 & & & & & & \\
\hline & -0.821 & 2,4,4-Trimethyl-2-cyclohexen-1-ol & 1054 & & & & & & \\
\hline & & & & 0.925 & 2-Methylbutanal & 736 & 0.803 & 3,4,4-Trimethyl-2-cyclohexen-1-one & 1082 \\
\hline & & & & 0.936 & 2-Methylpropanal & 710 & 0.820 & 2,2,6-Trimethylcyclohexanone & 1035 \\
\hline & & & & 0.954 & 3-Methylbutanal & 733 & 0.840 & Nonanal & 1107 \\
\hline & & & & & & & 0.850 & 4-Methylheptane & 788 \\
\hline & & & & & & & 0.853 & Hexanal & 812 \\
\hline & & & & & & & 0.875 & $\beta$-Ionone & 1508 \\
\hline & & & & & & & 0.918 & 2,6,6-Trimethyl-1-cyclohexene-1-acetaldehyde & 1261 \\
\hline & & & & & & & 0.927 & 3,5,5-Trimethyl-2-cyclohexen-1-ol & 1057 \\
\hline & & & & & & & 0.931 & $\alpha$-Humulene & 993 \\
\hline & & & & & & & 0.936 & 2,4,4-Trimethyl-2-cyclohexen-1-ol & 1054 \\
\hline & & & & & & & 0.936 & $\alpha$-Ionone & 1437 \\
\hline & & & & & & & 0.942 & $\begin{array}{l}\text { 3,3,5-Trimethyl-1,5-cyclohexadiene-1- } \\
\text { carbaldehyde }\end{array}$ & 1201 \\
\hline & & & & & & & 0.959 & $\beta$-Cyclocitral & 1223 \\
\hline \multirow[t]{5}{*}{ Red beet } & & & & 0.862 & 2-Methylpropanal & 710 & 0.812 & Heptane & 750 \\
\hline & & & & 0.880 & 3-Methylbutanal & 733 & 0.845 & Hexanal & 812 \\
\hline & & & & 0.892 & 2-Methylbutanal & 736 & & & \\
\hline & & & & 0.944 & Borane-dimethylsulfide & 705 & & & \\
\hline & & & & 0.973 & $\begin{array}{l}\text { 2-(1-Methylvinyl) } \\
\text { thiophene }\end{array}$ & 1005 & & & \\
\hline \multirow[t]{12}{*}{ Onion } & -0.894 & Methyl propyl disulfide & 930 & & & & -0.803 & Propyl disulfide & 1110 \\
\hline & -0.857 & Methyl propyl trisulfide & 1152 & & & & & & \\
\hline & -0.840 & 3,4-Dimethylthiophene & 903 & & & & & & \\
\hline & 0.918 & Dipropyl trisulfide & 1329 & 0.807 & Dimethyl trisulfide & 968 & 0.845 & (Z)-2-Heptenal & 956 \\
\hline & 0.925 & 1-Propanol & 710 & 0.813 & Benzeneacetaldehyde & 1045 & 0.865 & Heptanal & 902 \\
\hline & 0.956 & 1-Propanethiol & 720 & 0.873 & 3,4-Dimethylthiophene & 903 & 0.930 & 1-[(1-Methylethyl)thio]-pentane & 1091 \\
\hline & 0.965 & $\begin{array}{l}\text { 2-[2-(4-Methylphenyl)cyclopropanyl] } \\
\text { thiophene }\end{array}$ & 1601 & & & & 0.950 & (E)-2-octenal & 1060 \\
\hline & 0.967 & $\begin{array}{l}\text { 2-Mercapto-3,4-dimethyl-2,3- } \\
\text { dihydrothiophene }\end{array}$ & 1124 & 0.931 & Borane-dimethylsulfide & 705 & 0.962 & 2-Heptanone & 892 \\
\hline & 0.972 & 2,4-Dimethyl-thiophene & 1175 & 0.962 & 2-Methylbutanal & 736 & 0.983 & Hexanal & 812 \\
\hline & 0.987 & Allyl disulfide & 1129 & 0.974 & 3-Methylbutanal & 733 & & & \\
\hline & 0.992 & 3,5-Diethyl-1,2,4-trithiolane & 1344 & & & & & & \\
\hline & 0.992 & 1,5-Dithiocane & 1118 & & & & & & \\
\hline \multirow[t]{14}{*}{ Potato } & & & & & & & -0.803 & Heptanal & 902 \\
\hline & 0.810 & 2,4-Dimethyl-1-heptene & 846 & 0.848 & Methylene chloride & 707 & & & \\
\hline & 0.818 & 2-Propylidenecyclopentane & 1032 & 0.923 & 2-Ethylfuran & 751 & & & \\
\hline & 0.821 & 2-Methylpentene & 715 & 0.934 & 3-Methylbutanal & 733 & & & \\
\hline & 0.948 & Pentanal & 749 & 0.936 & 2-Pentylfuran & 992 & & & \\
\hline & 0.949 & Hexanal & 813 & 0.937 & 2-Methylbutanal & 736 & & & \\
\hline & 0.955 & 6-Methyl-3,5-heptadien-2-one & 860 & & & & & & \\
\hline & 0.957 & Octanal & 1004 & & & & & & \\
\hline & 0.966 & Pentyloxirane & 905 & & & & & & \\
\hline & 0.972 & 1-Chloro-pentane & 781 & & & & & & \\
\hline & 0.979 & 2 Octenal & 1060 & & & & & & \\
\hline & 0.979 & 3,5-Dimethyl-cyclohexanol & 1097 & & & & & & \\
\hline & 0.980 & (E)-2-hexenal & 857 & & & & & & \\
\hline & 0.981 & Decanal & 1207 & & & & & & \\
\hline
\end{tabular}

The volatiles that are selected in this work can be categorized into: (i) Strecker degradation aldehydes (2-methylpropanal, 3-methylbutanal and 2-methylbutanal); (ii) unsaturated fatty acid degradation products (heptane and hexanal); and (iii) sulfur-containing compounds (boranedimethylsulfide and 2-(1-methylvinyl)thiophene)). The observation with respect to Strecker degradation and unsaturated fatty acid degradation products is comparable to the discussion provided for pumpkin (Section 3.1), in which the formations of Strecker aldehydes were more pronounced by conventional thermal sterilization and unsaturated fatty acid degradation products were preferentially formed in HPHT sterilized samples. Sulfur-containing compounds were detected with higher levels after conventional thermal sterilization compared to HPHT sterilization. This observation may be explained by thermal decomposition of sulfur containing amino acids (e.g. S-methylmethionine) due to the exposure to the high temperature load during thermal processing (Vervoort et al., 2013; Kebede et al., 2013).

\subsection{Onion}

Onion is widely used in cooking and as a food ingredient. The major bioactive constituents in onions are sulfur compounds, in which most of them are in the form of cysteine derivatives. Something common to all 
Allium species is the enzyme alliinase, that catalyzes the hydrolysis of Salk(en)yl-L-cysteine sulfoxides (ACSOs) to produce pyruvate, ammonia and sulfenic acids. These spontaneous reactions result in the formation of major flavor compounds, e.g. mixture of sulfur-containing compounds (e.g. thiosulfinates, thiosulfonates, mono-, di- and tri-sulfides) as well as specific compounds such as the lachrymatory or tear factor (Colina-Coca, Gonzalez-Pena, Vega, de Ancos, \& Sanchez-Moreno, 2013). Similarly, in this work, the majority of the selected discriminant volatiles are sulfur-containing compounds. In addition, other compounds such as Strecker aldehydes, aliphatic aldehydes and alcohols were also selected. Intact onion cells have no characteristic flavor until cellular disruption. Only seconds after cell damage, rapid formation and release of flavor will take place. In the present work, the onions were sliced into standardized shapes before blanching. As this process could already trigger enzymatic cleavage of some flavor precursors, this might explain the formation of volatiles, such as dipropyl trisulfide, 1-propanol, 1-propanethiol and 2-[2-(4-methylphenyl)cyclopropanyl] thiophene, in blanched onions. Nevertheless, the course of sterilization seems to reduce the amount of these compounds as a lower amount of these compounds were detected in both HPHT and thermally processed onions. The possible explanation could be that the processing triggered degradation of these compounds into other reaction products or these highly volatile compounds were just lost during the course of processing. In contrast to the above observation, other volatiles such as methyl propyl disulfide and dimethyl trisulfide, were more detected after both sterilization processes. This means that in addition to the enzyme-catalyzed reaction pathways, processing at higher temperatures can also induce generation of characteristic processed onion sulfur-containing flavor compounds. The result with respect to the Strecker aldehydes (benzeneacetaldehyde, 2-methylbutanal and 3methylbutanal) and (oxidative) unsaturated fatty acid degradation aldehydes and ketones ((Z)-2-heptenal, heptanal, (E)-2-octenal, hexanal and 2-heptanone) is comparable to the observation described for pumpkin and red beet (Sections 3.1 and 3.2).

\subsection{Potato}

Raw potatoes have a high content of lipoxygenase, which catalyzes oxidative degradation of unsaturated fatty acids into volatiles upon cell disruption. Accordingly, volatiles, such as (E,Z)-2,4-decadienal, (E, $\mathrm{Z})$-2,6-nonadienal, and (E)-2-octenal and hexanal, were reported in freshly cut raw potatoes (Josephson \& Lindsay, 1987). Similarly, in this work, most of these aldehydes and hydrocarbons were detected in higher levels in blanched potato samples compared to thermal and HPHT sterilized samples. Even though the performed blanching condition (Section 2.1) was proven to be sufficient to reduce enzymatic activities below the detection limit (peroxidase test), enzyme-catalyzed reactions could already be triggered by the cellular disruption before blanching, triggering the formation these unsaturated fatty acid degradation products. Both conventional and HPHT sterilizations reduced the concentration of these volatiles, possibly, due to degradation into other reaction products and/or due to the volatile losses during processing. The volatile compounds which were detected at a higher concentration in the thermal class (2-ethylfuran, 2-pentylfuran, 3-methylbutanal and 2-methylbutanal) can be mainly linked to the Maillard reaction and Strecker degradation reaction. The observation that these reaction products are preferentially formed under conventional thermal sterilization is consistently observed in the other vegetables as well.

\section{Conclusion}

For the first time in literature, this work investigated the impact of HPHT sterilization in comparison to conventional thermal processing on four industrially-relevant vegetables, i.e. onion, potato, pumpkin and red beet. These vegetables were selected from different botanical families, edible plant parts and color groups, with the aim of investigating a wide range of chemical reactions. Despite the difference in the natural chemical composition, two common trends on processinduced reactions were observed among the vegetables: (i) in all vegetables, Strecker aldehydes were detected at a significantly higher amount in conventional thermal sterilized vegetables compared to HPHT sterilized vegetables; (ii) oxidative degradation reaction products of both unsaturated fatty acids (in pumpkin, red beet and onion) and carotenoids (in pumpkin) were detected at higher levels after HPHT sterilization. Therefore, it can be concluded that HPHT sterilization seems to reduce formation of Maillard reaction and Strecker degradation products and enhance oxidative degradation reaction products. The work's outcome is an important first step for setting up kinetic studies, to increase insight to the way individual process variables (e.g. pressure, temperature, time) are affecting selected quality-related chemical reactions.

Based on the results obtained, it is difficult to evaluate how the observed modification of the volatile fraction by HPHT processing compared to traditional processing will affect the overall flavor and taste. Accordingly, hypothesizing any industrial consequence is not straightforward. Fingerprinting only allows relative compound quantification, making any suggestion of absolute concentrations impossible. In addition, complex mixtures of compounds are responsible for flavor of each vegetable, and due to interactions, even a small change in the concentration of one compound may have major effects on the overall flavor. Therefore, it is obvious that future research steps should lead to (i) absolute quantification of the selected discriminant volatile compounds, using pure standards, and compare with concentration threshold values for the consumer (e.g. rejection); (ii) conduction of sensorial analysis, to understand how the observed process-induced changes will be appreciated.

In the context of this study and also related to our previous work, we would like to define 'processomics': a hypothesis-free screening tool to detect differences in process-induced chemical reactions and its reactions products. As proven by this work, it can be valuable to compare the process impact among different technologies immediately after processing and to zoom into relevant reaction pathways out of a complex network of chemical changes. Its potential to investigate quality changes during storage might be an interesting research topic for the near future.

\section{Acknowledgments}

This work was financially supported by KU Leuven Research Fund. One of the authors (T.G.) is a postdoctoral researcher funded by the Research Foundation Flanders (FWO) while S.P. was funded by the Agency for Innovation by Science and Technology in Flanders (IWT-Vlaanderen).

The authors thank Heidi Roba and Margot De Haes for their laboratory assistance and acknowledge and Agilent technologies, Diegem, Belgium for providing the Mass Profiler Professional (MPP) software.

\section{References}

Adebooye, O. C., Vijayalakshmi, R., \& Singh, V. (2008). Peroxidase activity, chlorophylls and antioxidant profile of two leaf vegetables (Solanum nigrum L. and Amaranthus cruentus L.) under six pretreatment methods before cooking. International Journal of Food Science and Technology, 43(1), 173-178.

Ahn, J., Balasubramaniam, V. M., \& Yousef, A. E. (2007). Inactivation kinetics of selected aerobic and anaerobic bacterial spores by pressure-assisted thermal processing. International Journal of Food Microbiology, 113(3), 321-329.

Colina-Coca, C., Gonzalez-Pena, D., Vega, E., de Ancos, B., \& Sanchez-Moreno, C. (2013) Novel approach for the determination of volatile compounds in processed onion by headspace gas chromatography-mass spectrometry (HS GC-MS). Talanta, 103, $137-144$.

de Heij, W., van Schepdael, L., Moezelaar, R., Hoogland, H., Matser, A., \& van den Berg, R. (2003). High pressure sterilization: maximizing the benefits of adiabatic heating. Food Technology, 57(3), 37-41.

De Roeck, A., Sila, D. N., Duvetter, T., Van Loey, A., \& Hendrickx, M. (2008). Effect of high pressure/high temperature processing on cell wall pectic substances in relation to firmness of carrot tissue. Food Chemistry, 107(3), 1225-1235. 
De Vleeschouwer, K., Van der Plancken, I., Van Loey, A., \& Hendrickx, M. E. (2010). The effect of high pressure-high temperature processing conditions on acrylamide formation and other Maillard reaction compounds. Journal of Agricultural and Food Chemistry, 58(22), 11740-11748.

Grauwet, T., Rauh, C., Van der Plancken, I., Vervoort, L., Hendrickx, M., Delgado, A., et al. (2012). Potential and limitations of methods for temperature uniformity mapping in high pressure thermal processing. Trends in Food Science E Technology, 23(2), 97-110.

Grauwet, T., Van der Plancken, I., Vervoort, L., Hendrickx, M., \& Van Loey, A. (2010). Protein-based indicator system for detection of temperature differences in high pressure high temperature processing. Food Research International, 43(1), 862-871.

Grauwet, T., Van der Plancken, I., Vervoort, L., Hendrickx, M., \& Van Loey, A. (2011). Temperature uniformity mapping in a high pressure high temperature reactor using a temperature sensitive indicator. Journal of Food Engineering, 105(1), 36-47.

Hill, V. M., Isaacs, N. S., Ledward, D. A., \& Ames, J. M. (1999). Effect of high hydrostatic pressure on the volatile components of a glucose-lysine model system. Journal of Agricultural and Food Chemistry, 47(9), 3675-3681.

Josephson, D. B., \& Lindsay, R. C. (1987). C4-heptenal - An influential volatile compound in boiled potato flavor. Journal of Food Science, 52(2), 328-331.

Kanasawud, P., \& Crouzet, J. C. (1990). Mechanism of formation of volatile compounds by thermal-degradation of carotenoids in aqueous-medium. 2. Lycopene degradation. Journal of Agricultural and Food Chemistry, 38(5), 1238-1242.

Kebede, B. T., Grauwet, T., Tabilo-Munizaga, G., Palmers, S., Vervoort, L., Hendrickx, M. et al. (2013). Headspace components that discriminate between thermal and high pressure high temperature treated green vegetables: Identification and linkage to possible process induced chemical changes. Food Chemistry, 141, 1603-1613.

Knorr, D., Froehling, A., Jaeger, H., Reineke, K., Schlueter, O., \& Schoessler, K. (2011). Emerging technologies in food processing. Molecular Nutrition \& Food Research, 2 203-235.

Matser, Ariette M., Krebbers, Bregje, van den Berg. Robert W. \& Bartels, Paul V. (2004) Advantages of high pressure sterilisation on quality of food products. Trends in Food Science E' Technology, 15(2), 79-85.

Meyer, R. S., Cooper, K. L., Knorr, D., \& Lelieveld, H. L. M. (2000). High-pressure sterilization of foods. Food Technology, 54(11), 67-72.

Mujica-Paz, H., Valdez-Fragoso, A., Samson, C. T., Welti-Chanes, J., \& Torres, J. A. (2011). High-pressure processing technologies for the pasteurization and sterilization of foods. Food and Bioprocess Technology, 4(6), 969-985.

Navarro, M., Verret, C., Pardon, R., \& EL Moueffak, A. (2002). Changes in volatile aromatic compounds of strawberry puree treated by high-pressure during storage. High Pressure Research, 22(3-4), 693-696.

Oey, Indrawati, Lille, Martina, Van Loey, Ann, \& Hendrickx, Marc (2008). Effect of high-pressure processing on colour, texture and flavour of fruit- and vegetable-based food products: A review. Trends in Food Science E' Technology, 19(6), 320-328.

Oey, Indrawati, Van der Plancken, Iesel, Van Loey, Ann, \& Hendrickx, Marc (2008). Does high pressure processing influence nutritional aspects of plant based food systems? Trends in Food Science \& Technology, 19(6), 300-308.
Porretta, S., Birzi, A., Ghizzoni, C., \& Vicini, E. (1995). Effects of ultra-high hydrostaticpressure treatments on the quality of tomato juice. Food Chemistry, 52(1), 35-41.

Ramaswamy, H. S., Shao, Y. W., \& Zhu, S. M. (2010). High-pressure destruction kinetics of Clostridium sporogenes ATCC 11437 spores in milk at elevated quasi-isothermal conditions. Journal of Food Engineering, 96(2), 249-257.

Rauh, C., Baars, A., \& Delgado, A. (2009). Uniformity of enzyme inactivation in a short-time high-pressure process. Journal of Food Engineering, 91(1), 154-163.

Reineke, K., Mathys, A., Heinz, V., \& Knorr, D. (2008). Temperature control for high pressure processes up to $1400 \mathrm{MPa}$. Journal of Physics: Conference Series, 121. http://dx.doi.org/10.1088/1742-6596/121/4/142012.

Schwartz, S. J., \& Vonelbe, J. H. (1980). Quantitative-determination of individual betacyanin pigments by high-performance liquid-chromatography. Journal of Agricultural and Food Chemistry, 28(3), 540-543.

Seo, J. S., Burri, B. J., Quan, Z. J., \& Neidlinger, T. R. (2005). Extraction and chromatography of carotenoids from pumpkin. Journal of Chromatography A, 1073(1-2), 371-375.

Siegmund, B., \& Murkovic, M. (2004). Changes in chemical composition of pumpkin seeds during the roasting process for production of pumpkin seed oil (part 2: volatile compounds). Food Chemistry, 84(3), 367-374.

Van der Plancken, I., Verbeyst, L., De Vleeschouwer, K., Grauwet, T., Heinio, R. L., Husband, F. A., et al. (2012). (Bio)chemical reactions during high pressure/high temperature processing affect safety and quality of plant-based foods. Trends in Food Science E Technology, 23(1), 28-38.

Verbeyst, L., Bogaerts, R., Van der Plancken, I., Hendrickx, M., \& Van Loey, A. (2012). Modelling of vitamin $C$ degradation during thermal and high-pressure treatments of red fruit. Food Bioprocess Technology, 6, pp. 1015-1023.

Vervoort, L., Grauwet, T., Kebede, B. T., Van der Plancken, L., Timmermans, R., Hendrickx, M., et al. (2012). Headspace fingerprinting as an untargeted approach to compare novel and traditional processing technologies: A case-study on orange juice pasteurisation. Food Chemistry, 134(4), 2303-2312.

Vervoort, L., Grauwet, T., Njoroge, D.M., Van der Plancken, I., Matser, A., Hendrickx, M., et al. (2013). Comparing thermal and high pressure processing of carrots at different processing intensities by headspace fingerprinting. Innovative Food Science \& Emerging Technologies, 18, 31-42.

Vervoort, L., Van der Plancken, L., Grauwet, T., Verlinde, P., Matser, A., Hendrickx, M., et al. (2012). Thermal versus high pressure processing of carrots: A comparative pilot-scale study on equivalent basis. Innovative Food Science E' Emerging Technologies, 15, 1-13.

Wilson, David R., Dabrowski, Lukasz, Stringer, Sandra, Moezelaar, Roy, \& Brocklehurst, Tim F. (2008). High pressure in combination with elevated temperature as a method for the sterilisation of food. Trends in Food Science \& Technology, 19(6), 289-299.

Yanwen, Shao, Songming, Zh. u., Ramaswamy, H., \& Marcotte, M. (2010). compression heating and temperature control for high-pressure destruction of bacterial spores; an experimental method for kinetics evaluation. Food and Bioprocess Technology, 3(1), 71-78. 